\title{
Intra- and inter-rater reliability of rectus femoris muscle thickness measured using ultrasonography in healthy individuals
}

\author{
Yosuke Takahashi', Yuji Fujino ${ }^{2 *}$, Kohei Miura' ${ }^{1}$ Ayumi Toida ${ }^{3}$, Tadamitsu Matsuda ${ }^{2}$ and Shigeru Makita ${ }^{4}$
}

\begin{abstract}
Background: Ultrasonography (US) is a feasible and accessible method for the measurement of skeletal muscle mass. This technique presents acceptable intra-rater reliability; however, there are a few reports on its inter-rater reliability. Additionally, relative reliability should equally be inspected to determine the presence of systematic errors. Therefore, this study aimed to investigate the intra- and inter-rater reliabilities and absolute reliability of rectus femoris muscle thickness as measured using US.

Methods: The participants included in our study comprised 12 healthy young men ( $26.5 \pm 3.9$ years. Rectus femoris muscle thickness was measured from the right side of the thigh using US by two trained physical therapists. Inter- and intra-rater reliabilities were determined using the intraclass correlation coefficient (ICC) $(1,1)$ and ICC $(2,1)$ methods, respectively. Absolute reliability was evaluated using Bland - Altman analysis. Additionally, we calculated the minimal detectable change at the $95 \%$ level of confidence $\left(\mathrm{MDC}_{95}\right)$.

Result: According to the results of the Bland - Altman analysis, no fixed or proportional errors were present. The ICC $(1,1)$ was 0.95 , and the ICC $(2,1)$ was 0.70 . The $\mathrm{MDC}_{95}$ values of rectus femoris thickness for the intra- and inter-rater reliabilities were $2.0 \mathrm{~mm}$ and $4.3 \mathrm{~mm}$, respectively.

Conclusions: In our study, intra- and inter-rater reliabilities were measured at "excellent" and "moderate" levels in the healthy individuals based on a previously defined scale. Moreover, we determined the measurement error for quantifying rectus femoris thickness. Therefore, the measurement of rectus femoris thickness using US could be considered applicable in clinical research.
\end{abstract}

Keywords: Skeletal muscle mass, Reliability, Ultrasonography, Rectus femoris muscle

\section{Background}

Loss of skeletal muscle mass is a major and well-known quantitative change associated with aging. Similarly, qualitative changes in skeletal muscle have been reported to correlate with aging, such as the preferential atrophy of type II muscle fibers [1, 2], increased intramuscular fat [3-5], and increased extracellular water volume relative to muscle volume [6]. The gold

\footnotetext{
*Correspondence: y.fujino.pb@juntendo.ac.jp

${ }^{2}$ Department of Physical Therapy, Faculty of Health Science, Juntendo

University, 3-2-12, Hongo, Bunkyo-ku, Tokyo 113-0033, Japan

Full list of author information is available at the end of the article
}

standard methods for skeletal muscle mass measurement are computed tomography (CT), magnetic resonance imaging (MRI), and dual-energy X-ray absorptiometry (DEXA) [7]. However, these techniques are not optimal for measuring qualitative changes in the skeletal muscle. Furthermore, they are not frequently used as they are costly and infer a risk of radiation exposure in a clinical setting. Ultrasonography (US) measurement is a more feasible and accessible method that can quantitatively and qualitatively evaluate muscles. Muscle thickness measured using US is similar to that measured using CT, MRI, or DEXA 
[8-11]. Therefore, US measurement has rapidly spread in the field of physical therapy.

The measurement of muscle thickness using US presents acceptable intra-rater reliability; however, there are a few reports on its inter-rater reliability [12-14]. Moreover, relative reliability cannot distinguish the type and extent of errors included in the measured values, as this statistical method is based on the assumption that only random errors occur. The measured error can be either systematic or random, and it is difficult to overcome systematic errors through repeated measurements. Hence, it is necessary to determine the presence of systematic errors.

\section{Materials and methods Aim and study design}

This study aimed to investigate the intra- and interrater reliabilities and absolute reliability of measuring rectus femoris muscle thickness using US. The present study design was a test-retest study (within-day and 1 week apart).

Table 1 Demographics of the participants

\begin{tabular}{lr}
\hline Characteristic & Total $(n=12)$ \\
\hline Age (years) & $26.5 \pm 3.9^{\mathrm{a}}$ \\
Height $(\mathrm{cm})$ & $174.3 \pm 0.1^{\mathrm{a}}$ \\
Weight $(\mathrm{kg})$ & $64.9 \pm 7.2^{\mathrm{a}}$ \\
BMI $\left(\mathrm{kg} / \mathrm{m}^{2}\right)$ & $21.3 \pm 1.9^{\mathrm{a}}$ \\
\hline
\end{tabular}

$B M I$ body mass index

a Values are presented as mean \pm standard deviation

\section{Participants}

A poster for research recruitment was posted in the first author's hospital to recruit participants for this study. Over a 12-month period (March 2017 to March 2018), 12 young male volunteers with a mean age of $26.5 \pm 3.9$ years (mean \pm standard deviation) participated in this study. Participant characteristics are shown in Table 1. The inclusion criteria included: (1) over 20 years old; (2) under 40 years old; (3) no orthopedic problem; (4) not hypersensitive to electrical stimulation; (5) able to provide informed consent. All participants received an explanation of the study purpose and provided written informed consent. The study was approved by the ethics committees of Saitama Medical University International Medical Center (16-188).

\section{Measurement of rectus femoris muscle thickness}

In the measurements performed using US, a transducer probe (UF-850XTD: Fukuda Denshi Co. Ltd., Tokyo, Japan) was used, as illustrated in Fig. 1b. Participants lay in the supine position with the knee extended and maintained this posture throughout the US measurement (Fig. 1). Rectus femoris muscle thickness was measured from the right side of the thigh. The ultrasound probe was placed on the anterior surface of the thigh, at the midpoint of the length between the anterior superior iliac spine and the upper border of the patella, referring to the measurement position of Pardo et al. [14]. The measurement sites were determined using a measuring tape and marked with a permanent marker. A circumferential mark was made perpendicularly at the midpoint of the long axis. The probe was placed at the top of this circumferential line (Fig. 2) and moved along the line until a suitable image was obtained, in which a parallel
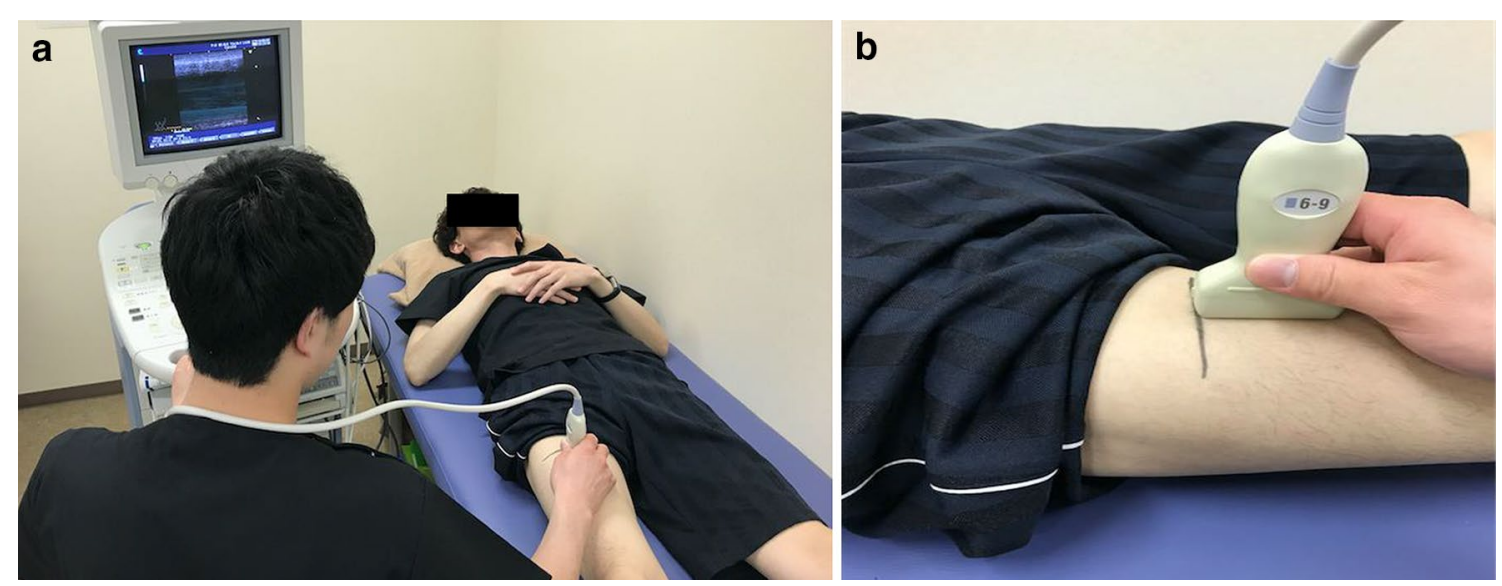

Fig. 1 Ultrasonography set-up in the measurement of skeletal muscle thickness. a Participant is depicted in a supine position while the user holds the ultrasound probe against the anterior surface of the thigh. $\mathbf{b}$ The exact position of the probe is illustrated. Muscle thickness was determined from the resulting ultrasound images, and the values from two independent raters were used to decipher the intra- and inter-rater reliabilities 


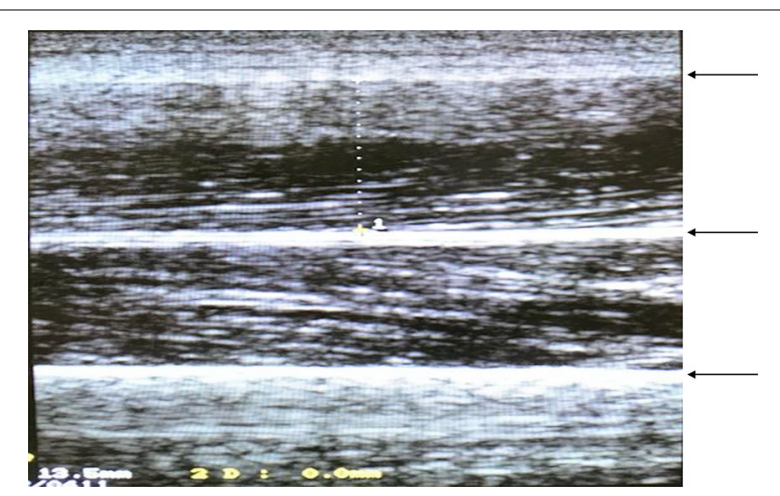

Fig. 2 Ultrasound of rectus femoris muscle. Ultrasound images of the muscular layers were obtained, and the muscle thickness of the rectus femoris was measured by drawing a perpendicular line from the border lines of the rectus femoris to the border lines of the vastus intermedius. On the screen of the US (ultrasonography) device, muscle thickness was calculated by setting the cursor to two points from the border lines of the rectus femoris to the border lines of vastus intermedius. Top arrow: rectus femoris muscle; middle arrow: vastus intermedius muscle; bottom arrow: femur

position of the three borderlines of the rectus femoris, vastus intermedius, and femur was achieved (Fig. 3). On the screen of the US device, muscle thickness was calculated by setting the cursor to two points from the border lines of the rectus femoris to the borderlines of vastus intermedius. The probe was maintained in this perpendicular position on the skin surface, and minimal contact pressure was applied during the measurements to obtain good-quality images.

Rectus femoris muscle thickness was measured by two trained physical therapists (A and B). To investigate the intra-rater reliability, the measurement was performed twice with an interval of 1 week by only rater A. To investigate the inter-rater reliability, the measurement was concurrently performed by two independent raters (A and $\mathrm{B}$ ); both raters were blinded to each other's results. We simultaneously focused on two or more measurements because we wanted to examine the interrater reliability by evaluating the concurrent measurement performed by two examiners.

\section{Data analysis}

The intraclass correlation coefficient (ICC) with 95\% confidence intervals (CI) was calculated to determine the relative reliability. Inter- and intra-rater reliabilities were determined using the ICC $(1,1)$ and ICC $(2$, 1) methods, respectively. The absolute reliability was evaluated using Bland-Altman analysis for obtaining intra- and inter-rater reliabilities. Furthermore, we calculated a confidence level of $95 \%$ of the average difference between the two measured values to detect the existence of fixed errors, and this was performed using the following formula:

$$
\bar{d} \pm t \times \sqrt{\frac{\mathrm{SDd}}{n}},
$$

where $\bar{d}$ is the average of 2 measurement values, $t$ is the degree of freedom in the case of $n_{-} 1, n$ is the sample size, and $\mathrm{SD}_{\mathrm{d}}$ is the standard error of $\bar{d}$

If the result did not contain 0 , we concluded that a fixed error was present, assuming that the measured values were distributed in a fixed direction [15]. Further, we calculated the $T$ value to inspect for the existence of a proportional error using the following formula:

$$
t=r \sqrt{\frac{n-2}{1-r^{2}}}
$$

$r$ is calculated using the correl function in Excel.

If the calculated $T$ value was higher than the $T$ value when the degree of freedom was $n-2$, and the level of significance was $5 \%$, we concluded that a proportional error was present, assuming that there was a significant correlation [15]. Additionally, we calculated the minimal detectable change at the $95 \%$ level of confidence $\left[\mathrm{MDC}_{95}=\right.$ standard error of measurement

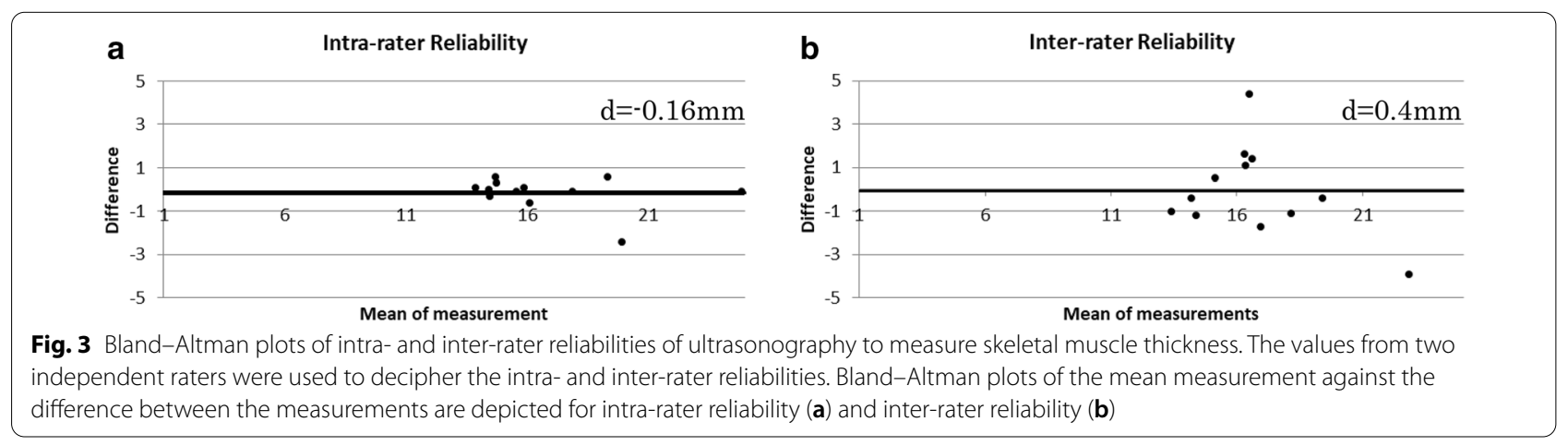


$(\mathrm{SEM}) \times 1.96 \times \sqrt{ } 2$ ], which is determined from the standard deviation of the difference (SDd): $\mathrm{SEM}=\mathrm{SDd} \times \sqrt{ } 2$. This represents the smallest change that can be interpreted as a real difference using the following formula:

$$
\mathrm{MDC}_{95}=1.96 \times \mathrm{SDd}
$$

Statistical analysis was performed using PASW Statistics ver. 18.0 (SPSS Japan Inc, Tokyo, Japan), with the level of significance set at $5 \%$.

\section{Results}

The two measurements obtained by rater A were $16.7 \pm 3.0 \mathrm{~mm}$ and $16.9 \pm 3.3 \mathrm{~mm}$, and the measurement obtained by rater B was $17.0 \pm 2.3 \mathrm{~mm}$ (Table 2). According to the results of the Bland - Altman analysis, no fixed or proportional errors were present. The ICC $(1,1)$ for the intra-rater reliability was 0.95 (95\% CI $0.856-0.987)$, and the ICC $(2,1)$ for the inter-rater reliability was 0.70 (95\% CI 0.225-0.901). The $\mathrm{MDC}_{95}$ values of rectus femoris thickness for the intra-rater and inter-rater reliabilities

Table 2 Measurement values taken by 2 independent raters

\begin{tabular}{llll}
\hline No. & $\begin{array}{l}\text { 1st assessment } \\
\text { Rater A (once) }(\mathrm{mm})\end{array}$ & $\begin{array}{l}\text { 2nd assessment } \\
\text { Rater A (twice) }(\mathrm{mm})\end{array}$ & Rater B (mm) \\
\hline 1 & 15.6 & 15.4 & 18.1 \\
2 & 14.5 & 14.4 & 19.5 \\
3 & 18.8 & 21.6 & 17.7 \\
4 & 17.6 & 18.4 & 16.3 \\
5 & 14.9 & 15.3 & 16.2 \\
6 & 15.5 & 14.4 & 14.0 \\
7 & 14.4 & 14.4 & 14.3 \\
8 & 19.5 & 18.9 & 20.0 \\
9 & 14.5 & 14.5 & 15.4 \\
10 & 24.3 & 24.5 & 20.5 \\
11 & 14.3 & 14.8 & 14.0 \\
12 & 16.8 & 16.3 & 17.5 \\
Average & 16.7 & 16.9 & 17.0 \\
SD & 3.0 & 3.3 & 2.3 \\
\hline
\end{tabular}

$S D$ standard deviation were $2.0 \mathrm{~mm}$ and $4.3 \mathrm{~mm}$, respectively (Table 3 ). The Bland-Altman plots were drawn (Fig. 4), and the differences in the scores were plotted against the mean scores of the measurements.

\section{Discussion}

In this study, we evaluated the intra- and inter-rater and absolute reliabilities of rectus femoris muscle measurement using US. Our findings support US as a useful tool for measuring rectus femoris muscle thickness in healthy young individuals. The primary use of analyses of healthy populations is to allow comparisons with pathological populations, and it follows, therefore, that measurement reliability in healthy populations is of importance. Few studies have compared MDC for intra- and inter-rater reliabilities; however, our study results indicated that rectus femoris muscle thickness should be measured by the same rater.

Based on the 95\% CI Koo et al. [16] reported that ICC values less than 0.5 , between 0.5 and 0.75 , between 0.75 and 0.9 , and greater than 0.9 indicate poor, moderate, good, and excellent reliability, respectively. The ICC values for the rectus femoris muscle thickness (intra-rater reliability $=0.99$, inter-rater reliability $=0.96$ ) presented here were similar to those reported by other studies [17, 18]. In this study, the ICCs of relative reliability was 0.95 for ICC $(1,1)$ and 0.70 for ICC $(2,1)$. ICC $(1,1)$ and ICC $(2,1)$, used to assess the intra- and inter-rater reliabilities, were at the "excellent" and "moderate" levels, respectively. Similarly, 95\% CI was $0.856-0.987$ for $\operatorname{ICC}(1,1)$ and $0.225-0.901$ for ICC $(2,1)$. ICC $(1,1)$ had "good" to "excellent" reliability; however, ICC $(2,1)$ significantly varied from "poor" to "excellent". Therefore, the intrarater reliability could be considered valid.

In addition, a systematic error was not detected in the absolute reliability results of the Bland-Altman analysis. The $\mathrm{MDC}_{95}$, a statistical parameter, can be used as a threshold to help users distinguish a real change from a measurement error for an individual participant. If the obtained value is equal to or less than the $\mathrm{MDC}_{95}$, then it is within the error range [19]. However, if the value is greater than the $\mathrm{MDC}_{95}$, it can be determined that a true change has occurred [19]. In this study, the $\mathrm{MDC}_{95}$ values for intra-rater and inter-rater reliabilities were

Table 3 The relative and absolute reliability results

\begin{tabular}{|c|c|c|c|c|c|c|}
\hline & \multirow{3}{*}{$\begin{array}{l}\text { Relative reliability } \\
\text { ICC (95\% CI) }\end{array}$} & \multicolumn{4}{|c|}{ Absolute reliability } & \multirow[t]{3}{*}{$\mathrm{MDC}_{95}(\mathrm{~mm})$} \\
\hline & & \multicolumn{2}{|l|}{ Fixed error } & \multicolumn{2}{|c|}{ Proportional error } & \\
\hline & & $95 \% \mathrm{Cl}$ & Result & $T$ value & result & \\
\hline Intra-rater reliability & $0.95(0.856-0.987)$ & $-0.8 \sim 0.4$ & No & -1.2 & No & 2.0 \\
\hline Inter-rater reliability & $0.70(0.257-0.901)$ & $-1.0 \sim 1.8$ & No & -1.6 & No & 4.3 \\
\hline
\end{tabular}

ICC intraclass correlation coefficient, Cl confidence interval, MDC95 minimal detectable change with $95 \% \mathrm{Cl}$ 
found to be $2.0 \mathrm{~mm}$ and $4.3 \mathrm{~mm}$, respectively, which is a useful index for judging the time course and therapeutic effect.

However, this study has some limitations. The amount of inward pressure on the patient caused by the transducer was not evaluated during the measurements. We believe that this is a possible reason why the inter-rater reliability was observed to be "moderate". In other words, it is necessary to consider the difference in the rater's measurement ability. These transducer conditions should have been precisely determined in the measurements obtained in the study. Another limitation is that only young males were enrolled in the study. Therefore, the influence of gender and age is unknown. Finally, a systematic error was not identified in this study. Thus, we should have calculated the appropriate repetition count to improve reliability. Further research is required to keep the amount of inward pressure caused by the transducer constant to ensure that interrater reliability is as good as intra-rater reliability. Similarly, more participants are needed to determine the effect of gender and age.

\section{Conclusions}

In conclusion, the intra-rater reliability and inter-rater reliability of the measurement of rectus femoris muscle thickness using US were mostly sufficient in healthy young men. Moreover, we determined the measurement error for rectus femoris thickness. Measuring the MDC is useful when verifying the degree of muscle atrophy and any therapeutic effects via the strengthening of muscles. Therefore, the measurement of rectus femoris muscle thickness using US could be considered applicable in clinical research.

\section{Abbreviations}

CT: Computed tomography; Cl: Confidence interval; DEXA: Dual-energy X-ray absorptiometry; ICC: Intraclass correlation coefficient; MRI: Magnetic resonance imaging; $\mathrm{MDC}_{95}$ : Minimal detectable change with $95 \% \mathrm{Cl}$; SD: Standard deviation; SEM: Standard error of measurement; US: Ultrasonography.

\section{Acknowledgements}

Not applicable.

\section{Authors' contributions}

YF designed the study. YT, YF and KM performed the data collection. YT and YF analyzed and interpreted the data and drafted the manuscript. AT contributed how to use the ultrasonography. TM reviewed and revised the manuscript. SM provided medical advice and overall study management. All authors read and approved the final manuscript.

\section{Funding}

This research received no specific funding.

\section{Availability of date and materials}

The datasets used and/or analyzed during the current study are available from the corresponding author on reasonable request.

\section{Declarations}

Ethics approval and consent to participate

All participants provided written informed consent, and the study was approved by the ethics committees of Saitama Medical University International Medical Center (16-188).

\section{Consent for publication}

The participants consented to the publication of their images.

\section{Competing interests}

Shigeru Makita received honorarium from Otsuka Pharmaceutical Co., Ltd. and Daiichi Sankyo Co., Ltd. and Fukuda Electronics Co., Ltd.

\section{Author details}

${ }^{1}$ Department of Rehabilitation Center, Saitama Medical University International Medical Center, 1397-1 Yamane, Hidaka, Saitama 350-1298, Japan. ${ }^{2}$ Department of Physical Therapy, Faculty of Health Science, Juntendo University, 3-2-12, Hongo, Bunkyo-ku, Tokyo 113-0033, Japan. ${ }^{3}$ Department of Central Examination, Saitama Medical University International Medical Center, 1397-1 Yamane, Hidaka, Saitama 350-1298, Japan. ${ }^{4}$ Department of Cardiac Rehabilitation, Saitama Medical University International Medical Center, Hidaka, Saitama 1397-1 Yamane350-1298, Japan.

Received: 1 July 2020 Accepted: 19 January 2021

Published online: 15 April 2021

\section{References}

1. Lexell J, Taylor CC (1988) Sjöström M (1998) What is the cause of the ageing atrophy? Total number, size and proportion of different fiber types studied in whole vastus lateralis muscle from 15 - to 83 -year-old men. J Neurol Sci 84:275-294

2. Evans WJ, Lexell J (1995) Human aging, muscle mass, and fiber type composition. J Gerontol A Biol Sci Med Sci 50:11-16

3. Tsubahara A, Chino N, Akaboshi K, Okajima Y, Takahashi H (1995) Age-related changes of water and fat content in muscles estimated by magnetic resonance (MR) imaging. Disabil Rehabil 17:298-304

4. Overend TJ, Cunningham DA, Paterson DH, Lefcoe MS (1992) Thigh composition in young and elderly men determined by computed tomography. Clin Physiol 12:629-640

5. Frantzell A, Ingelmark BE (1951) Occurrence and distribution of fat in human muscles at various age levels; a morphologic and roentgenologic investigation. Acta Soc Med Ups 56:59-87

6. DA YamadaY S, Nakamura E, Morimoto T, Kimura M, Oda S (2010) Extracellular water may mask actual muscle atrophy during aging. J Gerontol A Biol Sci Med Sci 65:510-516

7. Wells J (2006) Validity of methods used for the assessment of body components in children. Horm Res 66:58-64

8. Jolley SE, Bunnell AE, Hough CL (2016) ICU-acquired weakness. Chest 150:1129-1140

9. Kress JP, Hall JB (2014) ICU-acquired weakness and recovery from critical illness. N Engl J Med 370:1626-1635

10. Thomaes T, Thomis M, Onkelinx S, Coudyzer W, Cornelissen V, Vanhees L (2012) Reliability and validity of the ultrasound technique to measure the rectus femoris muscle diameter in older CAD-patients. BMC Med Imag 12:7

11. Dupont AC, Sauerbrei EE, Fenton PV, Shragge PC, Loeb GE, Richmond FJ (2001) Real-time sonography to estimate muscle thickness: comparison with MRI and CT. J Clin Ultrasound 29:230-236

12. Vijay H, Gopi CK, RohitK AD, Saurabh M, Maroof AK, Karan M, Anant M, Randeep G (2017) Intra- and inter-observer reliability of quadriceps muscle thickness measured with bedside ultrasonography by clinical care physicians. Indian J Crit Care Med 21:448-452

13. Silva CR, Costa AD, Rocha T, Lima DA, Nascimento TD, Moraes SR (2018) Quadriceps muscle architecture ultrasonography of individuals with type 2 diabetes: reliability and applicability. PLoS ONE 13:e0205724 
14. Pardo E, El Behi H, Boizeau P, Verdonk F, Alberti C, LescotT (2018) Reliability of ultrasound measurements of quadriceps muscle thickness in critically ill patients. BMC Anesthesiol 18:205-212

15. Ludbrook J (2002) Statistical techniques for comparing measurers methods of measurement: a critical review. Clin Exp Pharmacol Physiol 29:527-536

16. Koo TK, Li MY (2016) A guideline of selecting and reporting intraclass correlation coefficients for reliability research. J Chiropr 15:155-163

17. Thoirs K, English C (2009) Ultrasound measures of muscle thickness: intraexamine reliability and influence of body position. Clin Physiol Funct Imag 29:440-446
18. Tillquist M, Kutsogiannis DJ, Wischmeyer PE, Kummerlen C, Leung R, Stollery D, Karvellas CJ, Preiser JC, Bird N, Kozar R, Heyland DK (2014) Bedside ultrasound is a practical and reliable measurement tool for assessing quadriceps muscle layer thickness. J Parenter Enter Nutr 38:886-890

19. de Vet HC, Terwee CB, Knol DL, Bouter LM (2006) When to use agreement versus reliability measures. J Clinical Epidemiol 59:1033-1039

\section{Publisher's Note}

Springer Nature remains neutral with regard to jurisdictional claims in published maps and institutional affiliations.

\section{Submit your manuscript to a SpringerOpen ${ }^{\circ}$ journal and benefit from:}

- Convenient online submission

- Rigorous peer review

- Open access: articles freely available online

- High visibility within the field

- Retaining the copyright to your article

Submit your next manuscript at $\boldsymbol{\nabla}$ springeropen.com 